\section{New Ultra-Thin Pure Silicon Window Grids for Transmission Electron Microscopy Samples}

\author{
James Roussie \\ TEMwindows.com, Rochester, NY \\ jroussie@TEMwindows.com
}

\section{Introduction}

Traditional sample preparation supports for electron microscopy have usually been thin films of amorphous or holey carbon stretched over a metallic grid. More recently introduced formats include organic polymers over metallic grids or silicon nitride and silicon oxide membranes over silicon frames. Here, the application of a nanofabricated silicon membrane technology as a novel sample preparation support for electron microscopy is described. These new supports offer several unique characteristics, including nanoscale thickness and pores, which may improve imaging and analysis of materials and biological molecules.

Recently developed technology permits the fabrication of pure silicon membranes that are among the world's thinnest materials-only 5 to $15 \mathrm{~nm}$ thick. See Striemer et al., Nature (2007) 445: 749-751 for more details. These silicon membranes are also among the world's first membrane technologies to offer nanoscale pores of 5 to $50 \mathrm{~nm}$. This combination of characteristics is unique and not shared by the materials currently employed as sample supports. Moreover, it suggests that these silicon membranes could overcome many of the problems associated with current generation materials. For example, the holes of lacey and holey carbon grids (1-5 micron diameter) are incompatible with the size of most nanotubes and nanoparticles $(2-40 \mathrm{~nm})$. Obtaining background-free images of the entire nanostructure is rarely possible with this incompatible combination of sizes. Another significant issue is the inability to vigorously plasma clean samples on carbon grids. In some cases, this inability to remove organic contaminants can prevent high-resolution imaging of samples.

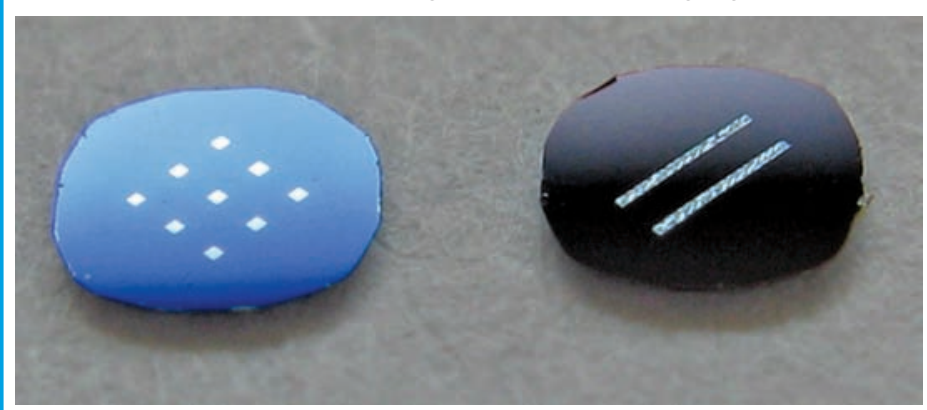

Figure 1: UltraSM Silicon TEM Windows Grids. Shown are grids with $2.9 \mathrm{~mm}$ diameter, $0.2 \mathrm{~mm}$ Si frames. A; An UltraSM ${ }^{\mathbb{R}}$ grid with nine $100 \times 100$ square windows. B: An UltraSM ${ }^{\circledR}$ grid with two $100 \times 2,000$ micron slots.

\section{Applications In TEM}

UltraSM $^{\circledR}$ Silicon TEM Window Grids incorporate this new membrane technology in a format convenient as a sample preparation support for transmission electron microscopy (TEM). Figure 1 show the two versions of UltraSM ${ }^{\circledR}$ grids-one with nine $100 \times$ 100 micron squares and one with two $100 \times 2,000$ micron slots. $\mathrm{UltraSM}^{\circledR}$ grids are available as continuous, amorphous Si films or as porous, nanocrystalline Si films (see Figure 2).

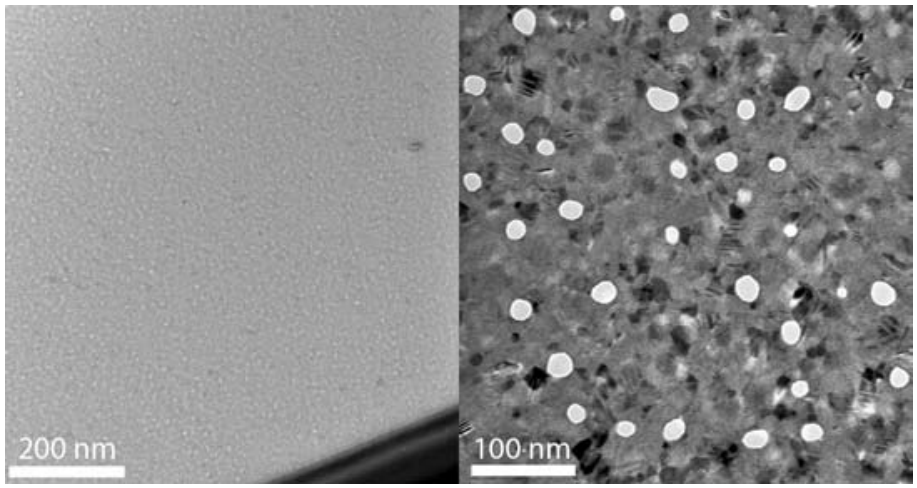

Figure 2: Amorphous and Porous UltraSM ${ }^{\circledR}$ Grids. (Left): A $5 \mathrm{~nm}$ thick amorphous UltraSM ${ }^{\mathbb{R}}$ grid. (Right): A $15 \mathrm{~nm}$ thick UltraSM ${ }^{\mathbb{R}}$ grid with 10-50 $\mathrm{nm}$ pores. Note that imaging the pores enhances the visibility of the nanocrystalline texture.

Porous UltraSM ${ }^{\circledR}$ grids contain approximately $10^{7}$ pores $/ \mathrm{mm}^{2}$ of 10-50 nm diameter ( $5 \%$ membrane porosity) as seen in Figure $2 \mathrm{~B}$. The pore formation process induces an inherent nanocrystallinity in these membranes and as a result, porous UltraSM ${ }^{\mathbb{R}}$ grids are polycrystalline. The $10-50 \mathrm{~nm}$ pores will support the suspension of nanoscale materials over open areas so that the materials can be imaged and analyzed without intervening background. Figure 3 shows a multi-walled carbon nanotube deposited on a $15 \mathrm{~nm}$ thick porous UltraSM $^{\circledR}$ grid. The multi-walled structure is clearly more distinguishable where the nanotube is over a pore.

Amorphous UltraSM ${ }^{\circledR}$ grids feature continuous, non-porous Si films of 5, 9 and $15 \mathrm{~nm}$ thickness. Compared to widely used carbon windows, nonporous UltraSM ${ }^{\circledR}$ TEM windows are more consistently thin, circumventing unpredictable variations in a window's thickness that would otherwise introduce additional background noise into images.

Due to their pure silicon composition, $\mathrm{UltraSM}^{\circledR}$ grids can withstand vigorous oxygen-plasma and UV-ozone cleaning. Both cleaning procedures reduce the surface contact angle well under 20 degrees, demonstrating the extent to which these grids can be cleaned. Removing organic contaminants, particularly from samples drawn up in organic and surfactant-containing solvents, is a difficult but critical step for high-resolution TEM. Residual contaminants can severely distort images due to increased sample charging. Conventional carbon grids cannot tolerate these cleaning procedures since they oxidize and degrade carbon films. The pure silicon composition of UltraSM ${ }^{\circledR}$ grids also increases their stability at high beam currents and annealing temperaturesproperties necessary for several of today's most demanding TEM techniques.

Elemental analysis studies by energy-dispersive X-ray (EDX) or electron energy loss spectroscopy (EELS) of samples containing nitrogen, oxygen and carbon also becomes much simpler when using a pure silicon grid. The background Si signal will be minimal and immediately distinguishable from the sample. Additionally, the polycrystalline nature of porous UltraSM $^{\circledR}$ grids yields a well-defined ring pattern that can serve as an internal reference standard when analyzing diffraction patterns. The obvious $<111>$ Si crystal lattice spacing at 3.14 Angstroms also provides an internal ruler against which the size of samples can be compared. 


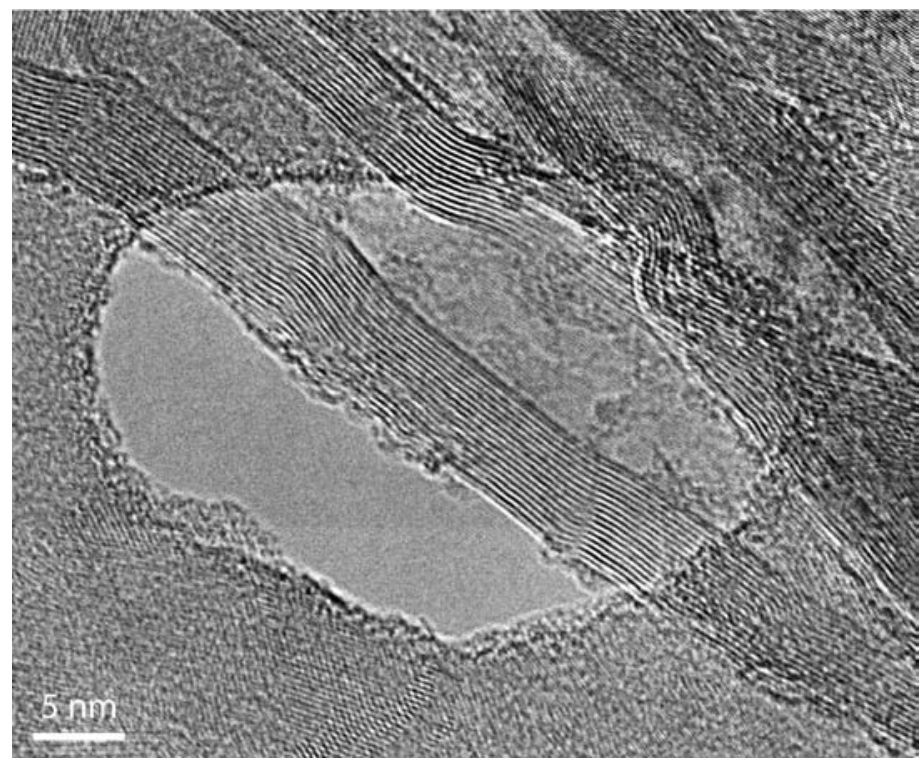

Figure 3: Multi-Walled Carbon Nanotubes Over Pores. Carbon nanotubes were deposited on $15 \mathrm{~nm}$ thick porous UltraSM ${ }^{\mathbb{R}}$ grids and imaged at 235,000-times magnification by TEM.

\section{Development And Fabrication Of Membranes}

$\mathrm{UltraSM}^{\circledR}$ membranes are fabricated as dies on Si wafers using modified semiconductor manufacturing techniques. Silicon wafers are initially patterned for the dies and a $\mathrm{Si}$ oxide layer is formed on one side of the wafer. Amorphous $\mathrm{Si}$ is laid on top of the oxide layer and an additional Si oxide layer deposited on top of the amorphous layer. Proprietary processing steps then form the membranes and create either the amorphous or porous membranes. The wafers are etched according to the initial pattern and the protective oxide layers removed, thereby suspending membranes on four sides by the rigid frame provided by the wafer. The dies are then punched out for use as TEM grids. Remarkably strong for only 50-70 silicon atoms, $15 \mathrm{~nm}$ thick UltraSM ${ }^{\circledR}$ membranes can support up to 1 atmosphere of pressure.

The UltraSM ${ }^{\circledR}$ membrane technology was first discovered at the University of Rochester in Rochester, New York. While studying the crystalline structure of Si thin films, researchers found it necessary to format the films in a manner suitable for TEM imaging. By transforming those films into membranes only $15 \mathrm{~nm}$ thick, they could more precisely image their intricate crystalline structures. These researchers soon realized that other investigators could benefit from this technology if formatted as TEM grids. Subsequently, a new company was formed and the patent-pending UltraSM ${ }^{\circledR}$ membrane technology exclusively licensed to SiMPore Inc. of Rochester, New York. In addition to pursuing other applications for these membranes, SiMPore is now distributing UltraSM ${ }^{\circledR}$ grids through TEMwindows.com.

\section{Acknowledgements}

Brian McIntyre, University of Rochester, and Thomas Gaborski and Nakul Nataraj, SiMPore Inc., prepared the images presented in this article.

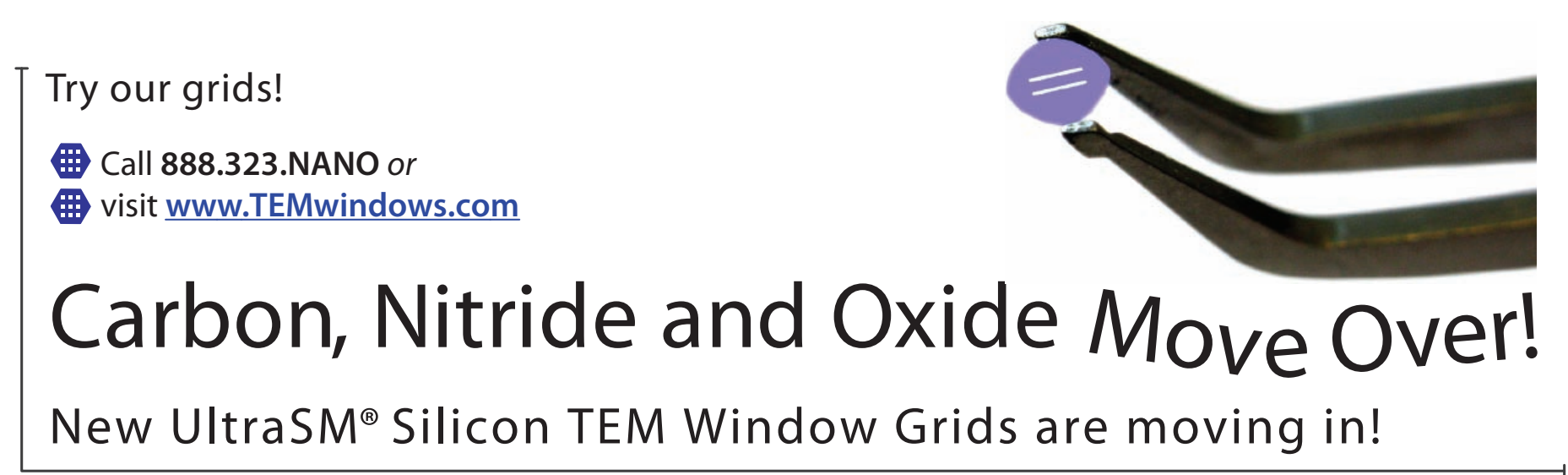

BACKGROUND FREE

- Suspend proteins, viruses or nanomaterials over countless 10 to $50 \mathrm{~nm}$ pores

NANOMETER THIN

- $15 \mathrm{~nm}$ nanoporous silicon fims

- 5, 9 and $15 \mathrm{~nm}$ continuous amorphous silicon films
PURE SILICON

- Analyze for carbon, nitrogen, and oxygen without interfering signals

STABLE

- Handle high beam currents and annealing temperatures

- Withstand extreme plasma cleaning 